\title{
A Review on Epidemiological Methods to Detect Untrue Information
}

\author{
Akanksha Mathur, C.P Gupta
}

\begin{abstract}
Online propagation of untrue information has been and is becoming an increasing problem. Understanding and modeling the diffusion of information on Online Social Networks (OSN's) of voluminous data is the prime concern. The paper provides the history of the epidemic spread and its analogy with untrue information. This paper provides a review of untrue information on online social networks and methods of detection of untrue information based on epidemiological models. Open research challenges and potential future research directions are also highlighted. The paper aimed at aiding research for the identification of untrue information on OSNs.
\end{abstract}

Keywords: Epidemics, Misinformation, Rumor Modelling, Social Networks

\section{INTRODUCTION}

$\mathrm{O}$ nline Social Networks (OSNs) are one of the most widely used Internet services worlds over. OSNs allow users to share ideas, thoughts, videos, and other types of media and have large-scale penetration among the population world over. OSNs differ characteristically in terms of (i) size of post, (ii) type of data allowed in the posts, (iii) method of propagation of posted information, (iv)method of collaboration, (v) data generated and interactions. With the increasing use of social media by people of all ages, various platforms such as Twitter, Facebook, Instagram, and Snapchat have become primary sources of news to people. This keeps a large portion of the population constantly updated on current events. The spread of information this way can be quite beneficial. People often act quickly without waiting to be informed by official news reports. However, this rapid spread of news can be harmful when false information breaks out similarly.

Untrue information has many forms and absolute classification of information to be true or untrue (i.e. fake) is not appropriate. Different classes intersect with each other as shown in Fig 1. Overlapping of these classes suggests that the truthfulness of information is dependent upon the context in which the information was posted. This suggests that binary classification of information (True/ fake) is inappropriate. In our opinion, it is more appropriate to assign a score to the

Manuscript received on August 26, 2021.

Revised Manuscript received on September 03, 2021.

Manuscript published on September 30, 2021.

Akanksha Mathur*, Research Scholar, Department of Computer Science and Engineering, Rajasthan Technical University, Kota (Rajasthan), India.

Prof. C.P Gupta, Professor, Department of Computer Science and Engineering, Rajasthan Technical University, Kota (Rajasthan), India.

(C) The Authors. Published by Blue Eyes Intelligence Engineering and Sciences Publication (BEIESP). This is an open access article under the CC BY-NC-ND license (http://creativecommons.org/licenses/by-nc-nd/4.0/) post reflecting its truthfulness.

*Correspondence Author

The characteristic of untrue information is, "the truth value is not verified at the time of posting". Spread of untrue information is a function of (i) size of user network (i.e. susceptible), (ii) number of users who believe the information to be true and retweet (i.e. infected), and (iii) elapsed time. Detecting untrue information poses many research challenges including diffusion time factors such as individual attributes, information properties, the strength of relations, etc. Social structures change with time, resulting in changes in diffusion patterns in dynamic situations. Other factors like its inconsistent vocabulary across the users and lack of availability of public datasets make the research incompatible to detect untrue information. Early detection of untrue information is the key to arresting the spread of such information. This paper examines the analogy between untrue and epidemic spread along with the introduction of various epidemic models from the literature. This gives a relationship of the epidemic with untrue information and open challenges in this direction.

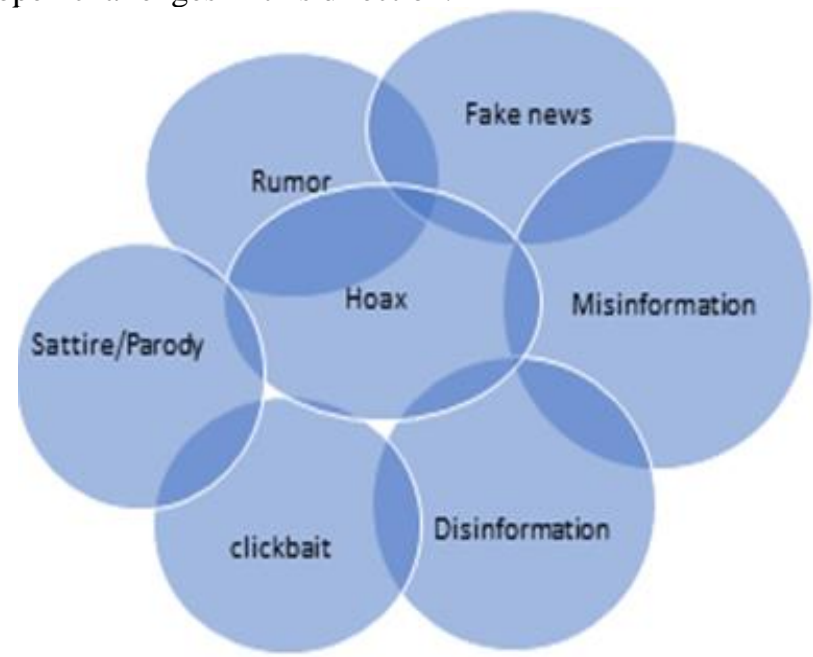

Fig 1. Categorization of Untrue Information

Social media is a data-generating and disseminating platform that generates massive amounts of data every second. Untrue information is a worldwide threat and a global issue. False information has real-life implications such that actions, views, and values can be altered. In general, inaccurate data is related to critical events and unexpected issues that are not confirmed by their sources. Untrue information identification is therefore extremely important. Moreover, the data generated by misinformation are unstructured, incomplete, and noisy, required the use of efficient methods to recognize untrue information. Research focusing on untrue information identification is still in its early stage.

Published By:

Blue Eyes Intelligence Engineering

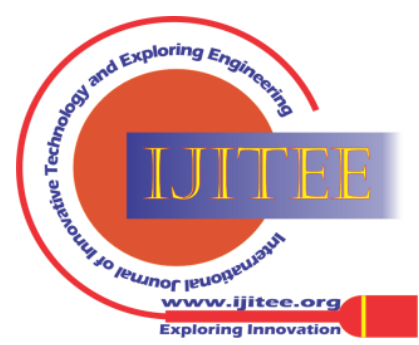




\section{A Review on Epidemiological Methods to Detect Untrue Information}

Most of the published work has neglected the following aspects: (i) Identifying structural features of untrue information; (ii) Ability to identify early stages of propagation; (iii) Developing an efficient and adaptable detection system for the analysis of false information.

\section{EPIDEMIC MODELS AND INFORMATION SPREAD}

There has been a significant amount of research on information diffusion in social networks, including epidemic models and rumor modeling techniques. A summary of these techniques is provided in the following section.

\section{A. Daley and Kendall Model}

"Epidemic-spread" refers to the transition from one state (i.e. susceptible) to another (i.e. infected/exposed), which is triggered by exposure to some (infection material) agent. The method isn't just for infectious diseases; it's a more general concept that can be used in a variety of situations. Susceptible, infected, removed, infectious material intermediate host, time of latency and diseases, and so on are some of the other process components. False information can make people vulnerable, and it can also make them resistant to others. After a while, a person infected with false information transmits it to others. As shown in Fig 2, this method produces well-explained rumor spread using a model with distinct epidemiological epochs involved in individual A infection and spread of B infection [1].

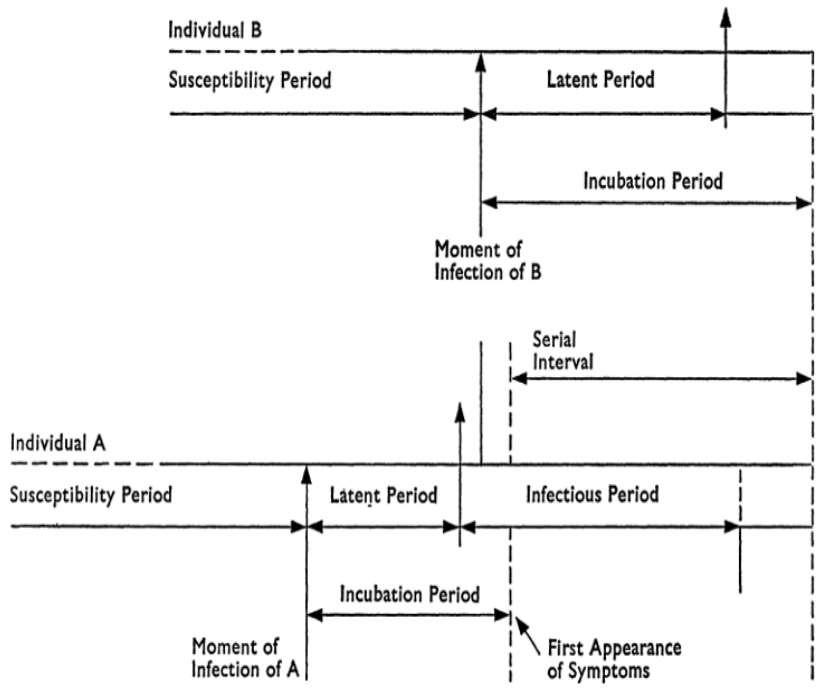

Fig 2. Relations Between Epidemiological Epochs

The time-specific definition of the various stages of infection is a unique feature of infectious disease epidemiology. The starting point is when a pathogen enters the host: this is known as the "exposure moment. "Following this, the pathogen usually moves to the desired tissue or target organ. This is the start of the infectious cycle of cellular and humored host defenses being activated. At this point, it's considered an "infection" (i.e. this can be symptomatic or asymptomatic). The time between exposure and infection is known as the latent phase because the pathogen is present in this state but the host shows no signs of infection. The "incubation phase" refers to the time between a diagnosis and the onset of clinical symptoms. The host can become infectious at any time during the infection (i.e. able to transmit the pathogen to other hosts). Figure 2 depicts the various periods.
When an active rumor spreader meets someone who has already heard about it, they are removed. If it also encounters a member of Class B, both will be transferred to Class C; however, if it encounters a member of Class $C$, only one will be transferred to $\mathrm{C}$. Acceptable transfer rates ( $\mathrm{Z}$ is a Stiffler number) are as follows:

$$
\begin{aligned}
& (X, Y, Z) \rightarrow(X-1, Y+1, Z) \quad X Y \\
& (X, Y, Z) \rightarrow(X, Y-2, Z+2) \quad \frac{1}{2} Y(Y-1) \\
& (X, Y, Z) \rightarrow(X, Y-1, Z+1) \quad Y Z \\
& (X, Y, Z) \rightarrow(X, Y, Z)-Y\left(X+\frac{1}{2}(Y-1)+Z\right)
\end{aligned}
$$

\section{B. Epidemic Models for Untrue Information Detection}

The diffusion of false information and the spread of an epidemic were quite similar. Time, relationship strength, information content, social factors, network structure, and other factors all have an impact on information dissemination. Researchers continued their development of the traditional model, creating new models such as the SI Model, the SIS Model[2], and the SIR model[3]. The SEIZ model takes a novel approach by including an Exposed state (E)[4].

The mapping of disease models with rumor models is very effective. Several papers investigate the properties of information dissemination in online social networks, with a focus on Twitter. The SIR model was supported by exposed(E) nodes and an SEIR[5] model was created with the addition of a new compartment. This is a dynamic method of disseminating information that takes into account the frequency with which users log in and friends interact. The results revealed that the frequency of logged-in users is directly proportional to the speed and range of data transmission. For an SEIR-supported one-layer social network, the S-SEIR[6] model was developed and sends user-based information. The SCIR model assumes if the user sends the message, that all followers have the contact status of 'C'[7]. For micro-blogs it provides a model for the online spread of the subject by adding a contact state (C). Moreover, the condition of the followers may change with a certain likelihood, transmission changes, or immune users after a certain period. The SIR-IR model is based on the SIR model, where user views were simulated and discontinued by adding mechanical infection recovery and checking on Facebook and Google[8]. The FSIR model considers the effect of the neighbor's on an individual in terms of diffusion information[9]. The information dissemination threshold is 0 and affects the diffusion of information. SIS-based ESIS model where the data sent by individuals expressed a kind of emotional information[10].

Furthermore, the DSIR and C-DSIR models introduced the double rumors dissemination mechanism through state vector expressions[11]. This breaks the constraints of many previous studies that pre-assume contradictory rumor contents or preset rumor spread priorities and makes the DSIR model more flexible and realistic in selecting the spreader's preferred rumor. 
Individual Susceptible Exposed Infectious Removed (iSEIR) is an abbreviation for Individual Susceptible Exposed Infectious Removed[12] which is an extension of the SEIR model for summarizing rumor-spreading behaviors of population interacting groups.

The simulation study results show that effective control of rumor spreading in the multiplex network requires efficient information flow management, which can be accomplished by setting appropriate immunization and spreading thresholds in individual behavior dynamics.

Compartmental models are a mathematical method for predicting and measuring the spread of infectious diseases. Misinformation is typically disseminated in the same way that a virus spreads. In viral transmission epidemics, each user is infected with viruses and has the potential to become infected.

Table- I: Epidemic Models for Untrue Information Detection

\begin{tabular}{|c|c|c|c|c|c|}
\hline $\begin{array}{c}\text { Scalable Model with } \\
\text { Year }\end{array}$ & Method & $\begin{array}{l}\text { Consider the } \\
\text { User's Different } \\
\text { Behaviors }\end{array}$ & $\begin{array}{c}\text { Dynamic } \\
\text { Infection and } \\
\text { Recovery Rates }\end{array}$ & $\begin{array}{l}\text { Performance } \\
\text { Metrics }\end{array}$ & Applications \\
\hline SEIR,2012 [5] & Insert Exposed node & No & No & $\begin{array}{l}\text { Distribution of nodes } \\
\text { density }\end{array}$ & $\begin{array}{l}\text { Determine the impact } \\
\text { factors: login frequency and } \\
\text { number of friends }\end{array}$ \\
\hline S-SEIR,2013 [6] & $\begin{array}{ll}\text { The significance } & \text { of } \\
\text { information } & \text { is } \\
\text { calculated } & \end{array}$ & Yes & No & $\begin{array}{l}\text { Distribution of } \\
\text { compartments-S, E, I, } \\
\text { and R }\end{array}$ & Simulate the diffusion \\
\hline SCIR, 2013 [7] & Insert contacted node & No & No & $\begin{array}{l}\text { Distribution of } \\
\text { compartments- I and R }\end{array}$ & $\begin{array}{l}\text { Represent the frequency } \\
\text { with which online topics } \\
\text { are spread }\end{array}$ \\
\hline irSIR, 2015 [8] & $\begin{array}{l}\text { Inserts dynamics } \\
\text { infection recovery }\end{array}$ & No & Yes & $\begin{array}{l}\text { Interaction with } \\
\text { real-world data }\end{array}$ & $\begin{array}{ll}\text { Describe } & \text { OSN } \\
\text { abandonment } & \end{array}$ \\
\hline FSIR,2015 [9] & $\begin{array}{l}\text { Consider } \\
\text { neighbors' actions }\end{array}$ & Yes & Yes & $\begin{array}{ll}\text { Interaction } & \text { with } \\
\text { real-world data } & \end{array}$ & $\begin{array}{l}\text { Determine the influencing } \\
\text { factors: information } \\
\text { number and friend number }\end{array}$ \\
\hline ESIS,2016 [10] & $\begin{array}{l}\text { Consider the emotional } \\
\text { impact of the } \\
\text { information }\end{array}$ & No & Yes & $\begin{array}{l}\text { Interaction with } \\
\text { real-world data to a } \\
\text { certain extent }\end{array}$ & $\begin{array}{l}\text { Determine the variables } \\
\text { that have an impact: } \\
\text { propagation probability and } \\
\text { transmission intensity }\end{array}$ \\
\hline $\begin{array}{l}\text { DSIR } \\
\text { C-DSIR,2018[11] }\end{array}$ & $\begin{array}{l}\text { Considering that only } \\
\text { two rumors are } \\
\text { spreading in the crowd } \\
\text { at the same time }\end{array}$ & Yes & yes & $\begin{array}{l}\text { The final state of the } \\
\text { stiflers' group and the } \\
\text { maximum value of the } \\
\text { spreaders density }\end{array}$ & $\begin{array}{l}\text { Developed a mechanism for } \\
\text { spreading double rumors } \\
\text { based on states-vector } \\
\text { expressions, and derived } \\
\text { model mean-field equations } \\
\text { to describe their dynamics. }\end{array}$ \\
\hline iSEIR ,2020[12] & $\begin{array}{l}\text { Assess } \\
\text { Individual-specific } \\
\text { behaviors }\end{array}$ & Yes & Yes & $\begin{array}{l}\text { Degree of fitting with } \\
\text { real data with time }\end{array}$ & $\begin{array}{l}\text { Detect effects of the } \\
\text { population size, the } \\
\text { individual distribution } \\
\text { density, and transition } \\
\text { probabilities }\end{array}$ \\
\hline
\end{tabular}

\section{OPEN RESEARCH CHALLENGES}

Extensive work has been reported to improve the reliability and trustworthiness of online content, but some key areas have gone unaddressed. To mitigate the spread of false information and its negative impact on society, the source must be identified quickly and in real-time. Real-time dataset collection, automatic rumor detection, and locating their origin are all difficult problems. The current research challenges are listed below:
- A scarcity of open datasets:

Because most research is conducted on customized datasets, the availability of reliable and standard datasets is critical. A benchmark comparison of different algorithms is not possible due to a lack of publicly available large-scale datasets.

- Cross-platform detection:

As people have accounts on various social networking sites, the propagation of incorrect information is difficult to manage.

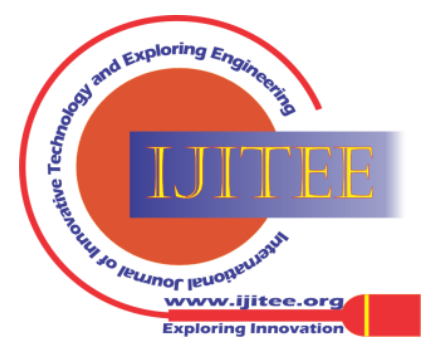




\section{A Review on Epidemiological Methods to Detect Untrue Information}

As a result, it has become a major issue to propagate false information from one site to another (i.e. cross-platform).

- Unsupervised models:

Current research is primarily based on supervised learning approaches. Because of the large amount of unlabeled data from social media, unsupervised models must be developed.

- Exploring in real-time:

Development of a web-based application for automated fact-checking and detection of fraudulent information in real-time.

- A platform that supports multiple languages:

The majority of the reported works concentrate solely on linguistic features in English language text. A multilingual platform for detecting fake news in popular and regional languages is highly desirable.

- Complex and dynamic network structure:

If we do the veracity classification task before the resolution, it becomes a prediction task and necessarily involves a huge portion of supporting evidence. This problem is exacerbated by the complex and dynamic network structure of social platforms.

- Cross-domain assessment:

The vast majority of existing approaches are solely focused on one mode of detection, either content, propagation, or style. Cross-domain analysis discovers unique non-variable traits and enables accurate detection of fraudulent content by integrating several aspects such as topic, website, language, images, and URL.

- Early detection:

Identifying fake news at an early stage is a difficult task. It is nearly impossible to change people's perceptions once they have become widespread and have gained the trust of users. As a result, it is highly desirable to take prompt action to mitigate it.

- False information detection in multimedia:

To detect fabricated and manipulated audio, images, and videos various techniques are used such as data analytics, computer vision, and image processing. Machine learning and deep learning algorithms will be useful in distinguishing between manipulated and fabricated multimedia.

\section{CONCLUSION}

Early warning can alert users to any potential risks associated with untrue information before they occur. The intervention against untrue information has the ability to eliminate users and so minimize the adverse effects of untrue information. The scope of this review is limited to epidemiological methods that predict whether given online information is true or false. This work can be expanded to include multi-level categorization techniques, such as machine learning and hybrid algorithms for detecting false information.

\section{REFERENCES}

1. D. J. Daley and D. G. Kendall, "Epidemics and Rumours [49]," Nature. 1964, DOI: 10.1038/2041118a0.

2. Wang Hao, Li Yiping, Feng Zhuonan, and Feng Ling, "ReTweeting Analysis and Prediction in Microblogs: An Epidemic Inspired Approach," CHINA Commun., 2013. and Sciences Publication

(C) Copyright: All rights reserved.
3. E. W. . S. Liu, D.; Yan, "M. Microblog information diffusion Simulation-based on sir model," J. Beijing Univ. Posts Telecommun, vol. 16. pp. 28-33, 2014.

4. F. Jin, E. Dougherty, P. Saraf, P. Mi, Y. Cao, and N. Ramakrishnan, "Epidemiological modeling of news and rumors on Twitter," in Proceedings of the 7th Workshop on Social Network Mining and Analysis, SNA-KDD 2013, 2013, DOI: 10.1145/2501025.2501027.

5. C. Wang, X. Y. Yang, K. Xu, and J. F. Ma, "SEIR-based model for the information spreading over SNS," Tien Tzu Hsueh Pao/Acta Electron. Sin., 2014, DOI: 10.3969/j.issn.0372-2112.2014.11.031.

6. H. L. and C. X. R. Xu, "Research on information dissemination model for social networking services," Int. J.Comput. Sci. Appl., vol. 1, no. 6, p. 2, 2013.

7. X. Ding, "Research on propagation model of public opinion topics based on SCIR in microblogging," Comput. Eng. Appl., vol. 20, no. 26, p. 51, 2015.

8. S. A. John, C.; Joshua, "Epidemiological modeling of online social network dynamics." 2014.

9. F. Y. L. X. Y. a. M. Y. Yang", "Automatic Detection of Rumor on Sina Weibo," in In Proceedings of the ACM SIGKDD Workshop on Mining Data Semantics, 2012.

10. S. C. and T. Y. Q. Wang, Z. Lin, Y. Jin, "Esis: Emotion-based spreader-ignorant-stifler model for information diffusion," Knowl.-Based Syst., vol. 46, no. 55, p. 81, 2015.

11. Y. Zan, "DSIR double-rumors spreading model in complex networks," Chaos, Solitons \& Fractals, vol. 110, pp. 191-202, May 2018, DOI: 10.1016/J.CHAOS.2018.03.021.

12. L. Di, Y. Gu, G. Qian, and G. X. Yuan, "A Dynamic Epidemic Model for Rumor Spread in Multiplex Network with Numerical Analysis $九$," 2020 .

\section{AUTHORS PROFILE}

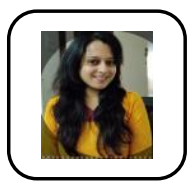

Akanksha Mathur is a research fellow at Rajasthan Technical University in Kota, Rajasthan, India. She post graduated with honors from Manipal University Jaipur with an MTech in Computer Science and Engineering. She has worked as a guest professor at Engineering College Bikaner. Big data analytics, social networks, machine learning, artificial intelligence, and neural networks are some of the current research areas.

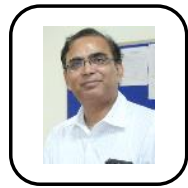

Prof. C.P Gupta is a computer science and engineering professor at Rajasthan Technical University in Kota. The current position is a continuation of my involvement with the former Engineering College in Kota, since 1990. He received his Ph.D. from the Faculty of Science at the University of Kota in Modeling of Nature Inspired Routing Algorithms for Wireless Sensor Networks with Mobile Sink. His current research focuses on information security and privacy, social network analysis, and network protocols.

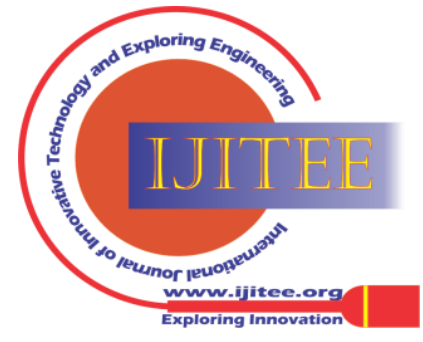

